\title{
A Simple and Computationally-Efficient SHE Technique Utilizing the Same Notch Angles for the Entire Modulation Range
}

\author{
Rakesh R, Student Member, IEEE, Siddavatam Ravi Prakash Reddy, Student Member, IEEE, \\ Mriganka Ghosh Majumder, Student Member, IEEE, Souradeep Pal, Student Member, IEEE, \\ K. Gopakumar, Fellow, IEEE, and L Umanand
}

\begin{abstract}
This paper proposes a novel and computationally efficient selective harmonic elimination (SHE) technique which eliminates the predefined lower order harmonics (till $19^{\text {th }}$ order) from the phase voltage while controlling its fundamental. In conventional SHE schemes, the notch angles need to be computed online for each frequency in order to eliminate the harmonics and control the fundamental value. This involves intensive online computations and the convergence to the correct notch angles is not guaranteed, resulting in incorrect fundamental and/or presence of lower order harmonics. In contrast to this, a SHE technique, that uses the same pre-computed notch angles for all modulation indices, is proposed in this paper, thereby significantly reducing the computational burden. Here, the control of fundamental voltage at different frequencies is ensured by the concept of phase shifting of two identical notched waveforms. This ensures precise control of fundamental voltage while completely eliminating the pre-defined lower order harmonics. Moreover, the proposed scheme exhibits linear control till 0.582 times the DC-link voltage compared to 0.577 times the DC-link voltage in case of space vector PWM. The proposed method is validated experimentally on an induction motor drive system.
\end{abstract}

Index Terms-3-phase inverter, Selective harmonic elimination, Low frequency pulse width modulation.

\section{INTRODUCTION}

Medium to high power converters are widely used in various applications like induction motor drives, permanent magnet synchronous motor drives, integration of renewable energy to grid, HVDC, electric traction etc [1]-[5]. At higher power levels, managing the thermal loss in semiconductor devices is an area of concern, as higher heat loss demands for bulky heat sinks and advanced cooling methods [6]. This demands the inverter to operate at lower switching frequencies. Modulation of the inverter using carrier based pulse width modulation (PWM) at lower switching frequencies generate undesired lower order harmonics and yield poor harmonic profile.

In this context, the elimination of lower order harmonic using selective harmonic elimination technique (SHE) comes to the fore. The ability to precisely eliminate the predefined lower order harmonics while operating the system at low frequency makes SHE superior to carrier based PWMs at higher power levels. The magnitude of each harmonic content is calculated mathematically from the pole voltage in terms of the notch angles using Fourier series, resulting in one transcendental equation for each harmonic. In traditional SHE technique, these transcendental equations are solved online using iterative numerical methods or random searching methods like Newton-Raphson algorithm, homotopy algorithm, genetic algorithm, particle swarm optimization technique etc [7]-[11]. The objective of the numerical method or random searching method is to eliminate the predefined harmonics and to obtain the required fundamental component for a particular modulation index. While computing real time on a digital signal processor (DSP), only limited iterations are possible and hence the convergence to correct notch angles may not be achieved, resulting in presence of lower order harmonics and/or incorrect fundamental component. Traditionally, an alternative method is used in which the notch angles for different modulation indices are computed offline and are then stored in a lookup table. This makes the scheme highly offline computationally intense. In addition, in a real time DSP, lookup tables for a certain amount of modulation indices can only be stored, and hence for any other modulation index the algorithm will select the values for the nearest modulation index, thus applying incorrect fundamental voltage.

Various works SHE techniques are reported in the literature [12]-[22]. In [12], the notch angles are computed through curve fitting approximation of the offline solution and this change is incorporated on the carrier waveform. However the carrier waveform has to be changed if the speed of operation is to be changed. In [13], the transcendental equation associated with each harmonic which are used in the optimization problem are converted to algebraic equations using Chebyshev polynomials. In [14] these set of algebraic equations are further reduced to a univalent polynomial using generalized Newton's identities. But this method involves solving of polynomial equations of higher powers for each modulation index, which makes it less attractive. Algebraic methods, without initial guesses, based on the Groebner bases and symmetric polynomial theory, are proposed in [15]-[17]. Here the order of polynomial is reduced to lower order using symmetric polynomial theory, thus making the scheme less complex. However, in these methods, solutions have to be obtained by solving the polynomials for different speeds of operation. In [23], a real-time implementation of SHE using generalized Hopfield neural network (GHNN) is proposed. This scheme suffers from the requirement of initial guesses and the presence of small traces of the harmonics that need to be eliminated in the phase voltage. SHE techniques based on artificial neural network (ANN) are presented in [18]-[20]. Here the accuracy of switching angle is dependent on the 
size of ANN. To get fairly accurate switching angles, the ANNs should be large and hence consume larger memory and implementation time. Four equation methods, which use four equations per harmonic to be eliminated is proposed in [21], [22]. Even though the equation to be solved are of lesser order, the complete elimination of predefined harmonics is not guaranteed in this method.

In this context, a computationally efficient SHE technique is proposed in this paper. The salient features of the scheme are as follows:

1) The same notch angles used for harmonic elimination at base speed of operation $(50 \mathrm{~Hz})$ are utilized for every other frequency of operation. This significantly reduces both the offline and online computations.

2) The fundamental component of the phase voltage required at different frequencies of operation is obtained by the sum of phase shifted identical notched waveforms. The profile of these identical notched waveforms is same as the pole voltage at $50 \mathrm{~Hz}$ and the profile will remain same irrespective of frequency of operation.

3) Since the identical notched waveforms are absent of predefined lower order harmonics, the addition of two such phase shifted waveforms will also be absent of lower order harmonics. This implies complete elimination of predefined harmonics along with precise control of fundamental component in phase voltage is ensured for any given frequency of operation even in a very low cost DSP.

4) The proposed scheme enables linear control in phase voltage to a maximum fundamental voltage of 0.582 times the DC-link, which is higher than the fundamental component obtained in linear modulation region by using space vector PWM (0.577 times the DC-link).

5) In the proposed scheme, the phase shift between the identical notched waveforms, which determines the amplitude and frequency of phase voltage is obtained at every sampling duration. This results in fast response of the scheme since adaptation for the change of frequency or amplitude of phase voltage can be done in the very next sampling duration.

\section{COMPUTATION OF NOTCH ANGLES FOR THE IDENTICAL} NOTCHED WAVEFORMS

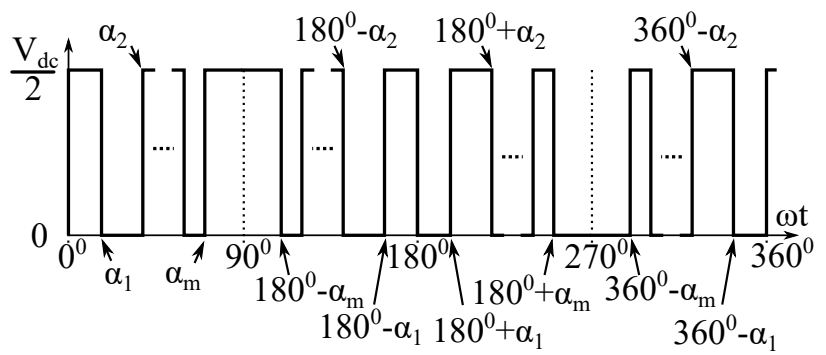

Fig. 1. Square waveform with predefined notches to eliminate lower order harmonics.

To eliminate lower order harmonics from a square waveform, predefined notches $\left(\alpha_{1}, \alpha_{2}, \ldots \alpha_{n}\right)$ per quarter are introduced in it. The $h^{\text {th }}$ harmonic component $\left(V_{h}\right.$, where $h=1$,
5,7 ...) in a notched waveform shown in Fig. 1 is given by (1).

$$
V_{h}=\frac{V_{d c}}{h \pi}\left[1+2 \sum_{j=1}^{m}\left[(-1)^{j} \cos \left(h * \alpha_{j}\right)\right]\right]
$$

Note that, since the resultant waveform (shown in Fig. 1) is quarter wave symmetric, even harmonics will be absent and hence they are not considered. In addition, since the scheme is implemented on a three phase system, where third harmonics is a zero sequence component, the effects of third harmonic component in the system, are also neglected.

Now, the objective is to eliminate the predefined harmonics from the notched square waveform and to maximize the fundamental component. In this work, the lower order harmonics till $19^{\text {th }}$ order (i.e. $5^{\text {th }}, 7^{\text {th }}, 11^{\text {th }}, 13^{\text {th }}, 17^{\text {th }}$ and $19^{\text {th }}$ ) are eliminated from motor phase voltage. So the objective function is given by (2)

$$
\begin{aligned}
& V_{5}^{2}+V_{7}^{2}+V_{11}^{2}+V_{13}^{2}+V_{17}^{2}+V_{19}^{2}=0 \\
& \text { and, maximize }\left(V_{1}\right)
\end{aligned}
$$

By offline computation using numerical methods, the values of $\alpha_{1}, \alpha_{2}, \ldots \alpha_{6}$ are obtained as given in (3).

$$
\begin{aligned}
& \alpha_{1}=7.8^{\circ}, \alpha_{2}=12.67^{\circ}, \alpha_{3}=23.09^{\circ}, \\
& \alpha_{4}=25.64^{\circ}, \alpha_{5}=38.13^{\circ}, \alpha_{6}=39^{\circ}
\end{aligned}
$$

The fundamental component of the notched waveform $\left(V_{1}\right)$ is obtained as $0.582 * V_{d c} / 2$. In this work, the notch angles obtained in (3) are used for any given frequency within the modulation range irrespective of the modulation index. The fundamental component is controlled for different modulation indices by the concept of phase shift, which will be discussed in the next section. This avoids iterative online computation to find the notch angles for every modulation index and makes the scheme implementation friendly. In conventional schemes, the notch angles have to be computed online by solving transcendental equations which makes the schemes computationally intensive. In addition, the elimination of lower order harmonics and obtaining of the required fundamental component are not guaranteed, as convergence of solution is not guaranteed in the limited iterations possible in online computation. In this work, one time calculation of notch angles by offline computation are sufficient for the elimination of predefined lower order harmonics throughout the modulation range.

\section{PRoposed SHE TECHNIQUe}

\section{A. Fundamental component control using phase shift}

Assume that there are two identical sinusoidal waveforms $\left(V_{s 1}\right.$ and $V_{s 2}$ ) having an amplitude of $V_{1}$ and are at the same frequency as shown in Fig. 2. These two sinusoids are shifted with respect to each other at an angle of $\theta$. Hence, these sinusoids $\left(V_{s 1}\right.$ and $\left.V_{s 2}\right)$ can be represented in polar form as given by (4), where $\gamma$ is an arbitrary angle.

$$
V_{s 1}=V_{1} \angle\left(\gamma-\frac{\theta}{2}\right) \quad \text { and } \quad V_{s 2}=V_{1} \angle\left(\gamma+\frac{\theta}{2}\right)
$$




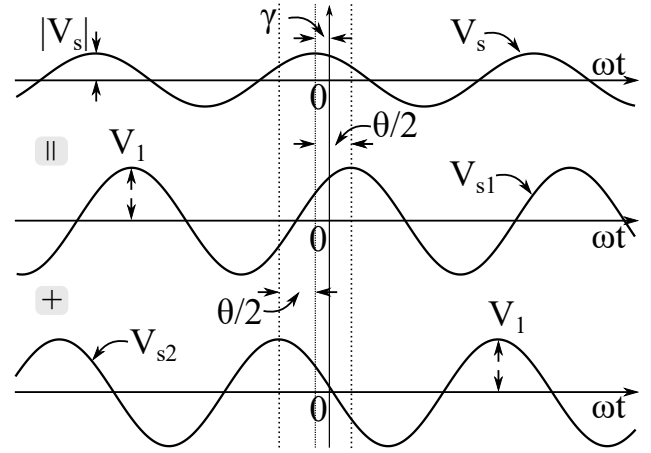

Fig. 2. Generation of the required fundamental component by the concept of sum of identical phase shifted waveforms

The summation of these two sinusoids will result in a third sinusoid $\left(V_{s}\right)$ with the same frequency and an amplitude given by (5) as shown in Fig. 2.

$$
V_{s}=V_{s 1}+V_{s 2}=2 V_{1} \cos \left(\frac{\theta}{2}\right) \angle \gamma
$$

Here it can been seen from (5) that the resultant sinusoid $\left(V_{s}\right)$ has an amplitude dependent on the phase shift between the two identical sinusoidal waveforms $V_{s 1}$ and $V_{s 2}$. In addition, the resultant sinusoid $\left(V_{s}\right)$ has a phase displacement $\gamma$, which is the arithmetic mean of the phase shifts of $V_{s 1}$ and $V_{s 2}$.

The phase shift $(\theta)$ required is obtained by rearranging (5) and is given by the relation (6).

$$
\cos \left(\frac{\theta}{2}\right)=\frac{\left|V_{s}\right|}{2 V_{1}}
$$

Here, $V_{s}$ can be visualized as the fundamental component of the phase voltage and the two identical sinusoids $V_{s 1}$ and $V_{s 2}$ can be visualized as the fundamental components of two identical phase shifted notched square waveform (obtained in previous section). Since the notched square waveform does not contain the predefined lower order harmonics, the summation of two such phase shifted waveform will also be free of the predefined lower order harmonics. The amplitude, frequency and phase angle of $V_{s}$ can be controlled by changing the phase shift (between $V_{s 1}$ and $V_{s 2}$ ), frequency and phase angle of $V_{s 1}$ and $V_{s 2}$ respectively. Hence for any frequency and voltage output in motor phase voltage, the same predefined notch angles can be used. At base speed of operation $(50 \mathrm{~Hz})$, the phase shift between the notched square waveforms are zero. This implies at $50 \mathrm{~Hz}$, the fundamental obtained has a magnitude of $2 V_{1}$ (by substituting $\theta=0$ in (6)) which equals to $0.582 * V_{d c}$, where $V_{d c}$ is the DC-link voltage. The fundamental component for any lower modulation indices can be obtained by linearly varying the value of $V_{s}$ in (6).

\section{B. Generation of phase shifted waveforms}

The output of control algorithm (scalar control, vector control etc.) gives the $\alpha \beta$ components $\left(V_{\alpha}\right.$ and $\left.V_{\beta}\right)$ of the reference voltage $\left(V_{r}\right.$, where $\left.V_{r}=V_{\alpha}+j V_{\beta}\right)$ which has to be applied across the 3-phase load terminals. The reference voltages along abc axes $\left(V_{A}, V_{B}\right.$ and $\left.V_{C}\right)$ are obtained using Clarke's transform (7).

$$
\left[\begin{array}{l}
V_{A} \\
V_{B} \\
V_{C}
\end{array}\right]=\frac{2}{3}\left[\begin{array}{cc}
1 & 0 \\
-0.5 & 0.866 \\
-0.5 & -0.866
\end{array}\right]\left[\begin{array}{l}
V_{\alpha} \\
V_{\beta}
\end{array}\right]
$$

Now, the objective is to generate two identical phase shifted sinusoidal waveforms $\left(V_{x 1}\right.$ and $V_{x 2}$, where $x=\mathrm{A}$, B or $\mathrm{C}$ phase) of same frequency as that of the required reference voltages in all the three phases. In each phase, one of the phase shifted waveforms $\left(V_{x 1}\right.$, where $x=\mathrm{A}, \mathrm{B}$ or $\mathrm{C}$ phase) should be lagging the reference voltage by $\theta / 2$ while the other $\left(V_{x 2}\right.$, where $x=\mathrm{A}$, B or C phase) leading by $\theta / 2$.

The phase shifted waveforms $V_{A 1}, V_{B 1}$ and $V_{C 1}$ will be three sinusoids having same amplitude and are shifted by $120^{\circ}$ with respect to each other. So, these waveforms can be also represented in $\alpha \beta$ plane as $V_{\alpha 1}$ and $V_{\beta 1}$. Since $V_{A 1}, V_{B 1}$ and $V_{C 1}$ are lagging the corresponding reference voltages $\left(V_{A}, V_{B}\right.$ and $V_{C}$ ) by $\theta / 2$, the $\alpha \beta$ components $V_{\alpha 1}$ and $V_{\beta 1}$ will also be lagging $V_{\alpha}$ and $V_{\beta}$ by $\theta / 2$ respectively as given by (8).

$$
\begin{aligned}
V_{\alpha 1}+j V_{\beta 1} & =\left(V_{\alpha}+j V_{\beta}\right) * e^{-j \theta / 2} \\
\Rightarrow V_{\alpha 1}+j V_{\beta 1} & =\left(V_{\alpha}+j V_{\beta}\right) *(\cos (\theta / 2)-j \sin (\theta / 2))
\end{aligned}
$$

Expanding (8) and splitting into real and imaginary parts gives (9).

$$
\begin{aligned}
& V_{\alpha 1}=V_{\alpha} * \cos (\theta / 2)+V_{\beta} * \sin (\theta / 2) \\
& V_{\beta 1}=-V_{\alpha} * \sin (\theta / 2)+V_{\beta} * \cos (\theta / 2)
\end{aligned}
$$

Similarly, the phase shifted waveforms $V_{A 2}, V_{B 2}$ and $V_{C 2}$ can be also represented in $\alpha \beta$ plane as $V_{\alpha 2}$ and $V_{\beta 2}$. Since $V_{A 2}, V_{B 2}$ and $V_{C 2}$ leads the corresponding reference voltages $\left(V_{A}, V_{B}\right.$ and $\left.V_{C}\right)$ by $\theta / 2$, the $\alpha \beta$ components $V_{\alpha 2}$ and $V_{\beta 2}$ will also be leading $V_{\alpha}$ and $V_{\beta}$ by $\theta / 2$ respectively as given by (10).

$$
\begin{aligned}
V_{\alpha 2}+j V_{\beta 2} & =\left(V_{\alpha}+j V_{\beta}\right) * e^{j \theta / 2} \\
\Rightarrow V_{\alpha 2}+j V_{\beta 2} & =\left(V_{\alpha}+j V_{\beta}\right) *(\cos (\theta / 2)+j \sin (\theta / 2))
\end{aligned}
$$

Expanding (10) and splitting into real and imaginary parts gives (11).

$$
\begin{aligned}
& V_{\alpha 2}=V_{\alpha} * \cos (\theta / 2)-V_{\beta} * \sin (\theta / 2) \\
& V_{\beta 2}=V_{\alpha} * \sin (\theta / 2)+V_{\beta} * \cos (\theta / 2)
\end{aligned}
$$

As explained in the previous section, the expression of phase shift can be obtained from (6) as a fraction of the amplitude of fundamental voltage required $\left(\left|V_{s}\right|\right)$ to twice the amplitude of fundamental component $\left(V_{1}\right)$ of the phase shifted waveform. The expression for $\left|V_{s}\right|$ can be obtained from $V_{\alpha}$ and $V_{\beta}$ as given in (12), where scaling factor of $2 / 3$ is obtained from $\alpha \beta$ to abc conversion.

$$
\left|V_{s}\right|=(2 / 3) * \sqrt{V_{\alpha}^{2}+V_{\beta}^{2}}
$$

By substituting the value of $\left|V_{s}\right|$ from (12) in (6) modifies the expression of phase shift to (13) 


$$
\cos \left(\frac{\theta}{2}\right)=\frac{\sqrt{V_{\alpha}^{2}+V_{\beta}^{2}}}{3 V_{1}}
$$

It can be seen from (9) and (11) that, finding the $\alpha \beta$ components of the phase shifted waveforms requires only multiplication with $\cos (\theta / 2)$ and $\sin (\theta / 2)$ (14). However, the value of $\cos (\theta / 2)$ and $\sin (\theta / 2)$ are obtained from (13), (14) and no trigonometric operation is required, which makes the scheme more implementation friendly.

$$
\sin \left(\frac{\theta}{2}\right)=\sqrt{1-\cos ^{2}(\theta / 2)}
$$

\section{Generation of notches}

Using (9) and (11) the phase shifted notched waveforms will be obtained in all the three phases, which will be discussed hereinafter. By applying Clarke's transformation (7), the $\alpha \beta$ components ([$\left.V_{\alpha 1}, V_{\beta 1}\right]$ and $\left.\left[V_{\alpha 2}, V_{\beta 2}\right]\right)$ of the phase shifted waveforms are converted to its abc components ( $\left[V_{A 1}, V_{B 1}\right.$, $\left.V_{C 1}\right]$ and $\left.\left[V_{A 2}, V_{B 2}, V_{C 2}\right]\right)$ respectively. These waveforms provide the phase and frequency of the phase shifted notched square waveforms.

Since $V_{x y}$ (where $x=\mathrm{A}, \mathrm{B}, \mathrm{C}$ and $y=1,2$ ) is a sinusoidal waveform, $V_{x y}$ is represented as (15), where $\alpha$ is in first quadrant $(0 \leq \alpha<90)$ and $\left|V_{s}\right|$ is as given in (12).

$$
V_{x y} /\left|V_{s}\right|=\sin (\alpha)
$$

Consider a notch has to be introduced in the square waveform between $\alpha_{1}$ and $\alpha_{2}$, where $0 \leq \alpha_{1} \leq \alpha_{2} \leq 90^{\circ}$. Then for an arbitrary angle $\alpha$ lying in the first quadrant will satisfy the condition $\alpha_{1} \leq \alpha \leq \alpha_{2}$ if and only if (16) is satisfied.

$$
\sin \alpha_{1} \leq \sin \alpha \leq \sin \alpha_{2}
$$

By substituting (15) on (16), the inequality changes to (17).

$$
\begin{aligned}
& \sin \alpha_{1} \leq V_{x y} /\left|V_{s}\right| \leq \sin \alpha_{2} \\
\Rightarrow & \left|V_{s}\right| \sin \alpha_{1} \leq V_{x y} \leq\left|V_{s}\right| \sin \alpha_{2}
\end{aligned}
$$

Since the resultant notched square waveform is quarter wave symmetric, if there is a notch angle $\alpha_{1}$ in the first quadrant $\left(0 \leq \alpha_{1}<90\right)$, the same notch will be repeated in $180^{\circ}-\alpha_{1}$, $180^{\circ}+\alpha_{1}$ and $360^{\circ}-\alpha_{1}$ in second, third and fourth quadrants respectively. Hence the relation given by (18) hold good.

$$
\begin{aligned}
\sin \left(\alpha_{1}\right) & =\left|\sin \left(180^{\circ}-\alpha_{1}\right)\right|=\left|\sin \left(180^{\circ}+\alpha_{1}\right)\right| \\
& =\left|\sin \left(360^{\circ}-\alpha_{1}\right)\right|=\left|V_{x y}\right| /\left|V_{s}\right|
\end{aligned}
$$

So, the condition for a quarter wave symmetric notch lying in any quadrant in terms of first quadrant angles $\alpha_{1}$ and $\alpha_{2}$ is obtained by modifying (17) by substituting (18) in (16) as (19).

$$
\begin{aligned}
& \sin \alpha_{1} \leq\left|V_{x y}\right| /\left|V_{s}\right| \leq \sin \alpha_{2} \\
& \Rightarrow\left|V_{s}\right| \sin \alpha_{1} \leq\left|V_{x y}\right| \leq\left|V_{s}\right| \sin \alpha_{2}
\end{aligned}
$$

Similarly, notches has to be introduced between the angles $\left[\alpha_{3}\right.$, $\left.\alpha_{4}\right]$ and $\left[\alpha_{5}, \alpha_{6}\right]$. The notched square waveforms $V_{x y, \text { notch }}$ (where $x=\mathrm{A}, \mathrm{B}, \mathrm{C}$ and $y=1,2$ ) are generated by using (19) as shown in Table I.

TABLE I. GENERATION OF NOTCHED SQUARE WAVEFORM $\left(V_{x y, \text { notch }}\right)$

\begin{tabular}{|c|c|c|}
\hline \multirow{2}{*}{ Condition } & \multicolumn{2}{|c|}{$\begin{array}{c}\text { Value of } \\
V_{x y, n o t c h}\end{array}$} \\
\cline { 2 - 3 } & $\begin{array}{c}\text { +ve half } \\
\text { of } V_{x y}\end{array}$ & $\begin{array}{c}\text {-ve half } \\
\text { of } V_{x y}\end{array}$ \\
\hline$\left|V_{s}\right| \sin \alpha_{1} \leq\left|V_{x y}\right| \leq\left|V_{s}\right| \sin \alpha_{2}$ & 0 & $V_{d c} / 2$ \\
\hline$\left|V_{s}\right| \sin \alpha_{3} \leq\left|V_{x y}\right| \leq\left|V_{s}\right| \sin \alpha_{4}$ & 0 & $V_{d c} / 2$ \\
\hline$\left|V_{s}\right| \sin \alpha_{5} \leq\left|V_{x y}\right| \leq\left|V_{s}\right| \sin \alpha_{6}$ & 0 & $V_{d c} / 2$ \\
\hline For all other conditions & $V_{d c} / 2$ & 0 \\
\hline
\end{tabular}

Note: $x=\mathrm{A}, \mathrm{B}, \mathrm{C}$ and $y=1,2$

$\alpha_{1}, \alpha_{2}, \alpha_{3}, \alpha_{4}, \alpha_{5}, \alpha_{6}$ are the notch angles obtained in (3)

D. Generation of required pole voltage and power circuit realization

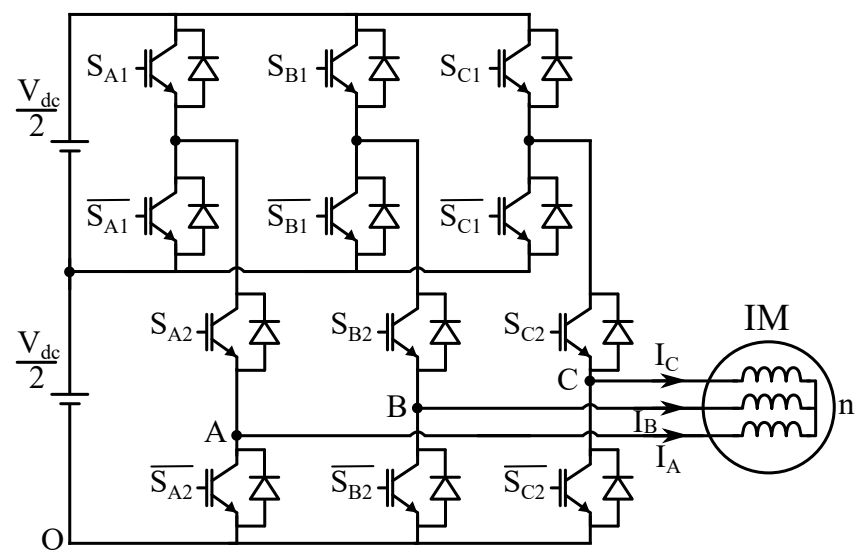

Fig. 3. Inverter topology used for implementing the proposed scheme

Now, for any frequency and amplitude of motor phase voltage, there are two phase shifted notched square waveforms $\left(V_{x 1, \text { notch }}\right.$ and $V_{x 2, \text { notch }}$, where $\left.x=\mathrm{A}, \mathrm{B}, \mathrm{C}\right)$ in each phase. The required pole voltage is the arithmetic sum of these phase shifted notched waveforms as explained in section III-A. At an arbitrary instant, the pole voltage $\left(V_{x O}=V_{x 1, \text { notch }}+\right.$ $V_{x 2, \text { notch }}$, where $x=\mathrm{A}, \mathrm{B}, \mathrm{C}$ ) can have three values $0, V_{d c} / 2$ and $V_{d c}$. So the scheme can be realized on any 3-Level inverter. The scheme is implemented on the inverter topology shown in Fig. 3. The topology consists of two set of complementary pair of switches $\left(\left[S_{x 1}, \overline{S_{x 1}}\right]\right.$ and $\left[S_{x 2}, \overline{S_{x 2}}\right]$, where $x=\mathrm{A}, \mathrm{B}$, C) in each phase. The pole voltage $\left(V_{x O}\right.$, where $\left.x=\mathrm{A}, \mathrm{B}, \mathrm{C}\right)$ and status of switches $\left[S_{x 1}, S_{x 2}\right]$ for different values of phase shifted notched square waveforms $\left(V_{x 1, \text { notch }}\right.$ and $\left.V_{x 2, \text { notch }}\right)$ are shown in Table II.

\section{E. Summary of the algorithm}

The proposed SHE algorithm is explained systematically in brief using the following steps:

1) Step 1: Input the $\alpha \beta$ components $\left(V_{\alpha}\right.$ and $\left.V_{\beta}\right)$ of reference vector. 
TABLE II. POLE VOLTAGE FROM PHASE SHIFTED NOTCHED SQUARE WAVEFORMS

\begin{tabular}{|c|c|c|c|c|}
\hline$V_{x 1, \text { notch }}$ & $V_{x 2, \text { notch }}$ & $V_{x O}$ & $S_{x 1}$ & $S_{x 2}$ \\
\hline 0 & 0 & 0 & OFF & OFF \\
\hline 0 & $V_{d c} / 2$ & $V_{d c} / 2$ & OFF & ON \\
\hline$V_{d c} / 2$ & 0 & $V_{d c} / 2$ & OFF & ON \\
\hline$V_{d c} / 2$ & $V_{d c} / 2$ & $V_{d c}$ & ON & ON \\
\hline
\end{tabular}

Note: $x=\mathrm{A}, \mathrm{B}, \mathrm{C}$ phase

2) Step 2: Compute the magnitude of reference vector $\left(\left|V_{s}\right|\right)$ in using $(12)$.

3) Step 3: Calculate the cosine and sine of phase shift $(\cos (\theta / 2)$ and $\sin (\theta / 2))$ required for the phase shifted waveforms using (13) and (14).

4) Step 4: Compute the $\alpha \beta$ components of the phase shifted waveforms ([$\left.V_{\alpha 1}, V_{\beta 1}\right]$ and $\left.\left[V_{\alpha 2}, V_{\beta 2}\right]\right)$ using (9) and (11).

5) Step 5: Obtain the phase shifted waveforms in abc phases $\left(\left[V_{A 1}, V_{B 1}, V_{C 1}\right]\right.$ and $\left.\left[V_{A 2}, V_{B 2}, V_{C 2}\right]\right)$ from $\left[V_{\alpha 1}\right.$, $\left.V_{\beta 1}\right]$ and $\left[V_{\alpha 2}, V_{\beta 2}\right]$ using Clarke's transformation given in (7).

6) Step 6: Obtain the phase shifted notched waveforms ([ $V_{A 1, \text { notch }}, V_{B 1, \text { notch }}, V_{C 1, \text { notch }}, V_{A 2, \text { notch }}, V_{B 2, \text { notch }}$, $\left.V_{C 2, \text { notch }}\right]$ ) from phase shifted waveforms ([ $V_{A 1}, V_{B 1}, V_{C 1}$, $\left.\left.V_{A 2}, V_{B 2}, V_{C 2}\right]\right)$ using Table I.

7) Step 7: Compute the pole voltages from phase shifted notched waveforms ([$V_{A 1, \text { notch }}, V_{B 1, \text { notch }}, V_{C 1, \text { notch }}$, $\left.\left.V_{A 2, \text { notch }}, V_{B 2, \text { notch }}, V_{C 2, \text { notch }}\right]\right)$ and apply proper switching states to the inverter according to the information given in Table II.

It can be noted that while going from Step 1 to Step 7, no iterative steps were required, and hence saving the computation time. This algorithm will work for the full modulation range by using the same predefined notch angles and the fundamental is controlled precisely by the concept of phase shift. It can be also noted that, the algorithm does not use trigonometric functions and the cosine and sine functions $(\cos (\theta / 2)$ and $\sin (\theta / 2))$ used in the algorithm is obtained from simple algebra using (13) and (14). The precise control of fundamental component with the elimination of predefined lower order harmonics is achieved without solving transcendental equations in online (like in the case of conventional SHE schemes), making the scheme attractive.

\section{RESULTS AND DISCUSSION}

\section{A. Hardware Implementation details}

The control logic is implemented on TMS320F28334 DSP from Texas Instruments. The reference frequency of operation is taken as the input of DSP and real time switching states are delivered as the output. The DSP is operated at a sampling frequency of $50 \mathrm{kHz}$ and at each sampling interval the phase shift as well as the switching states are determined. These switching states are then passed through a complementary and dead time generation circuitry. The dead time incorporated complementary signals are then passed through the gate driver. ISO5451 IC is used as the core IC of the gate driver board.

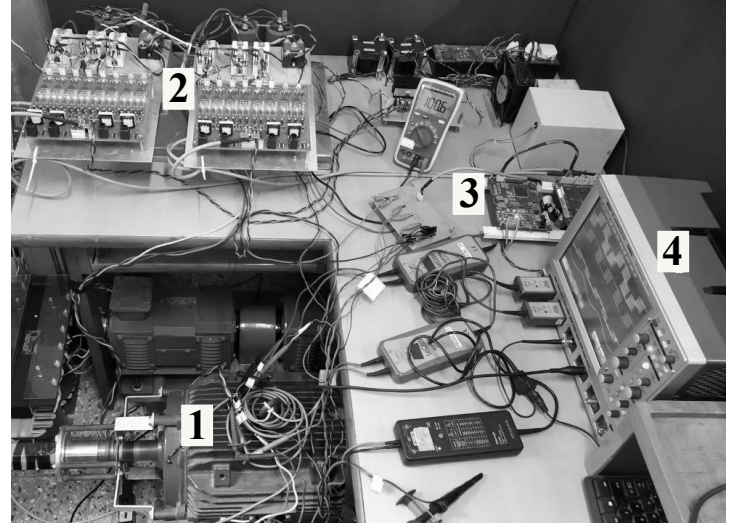

Fig. 4. Photograph of the experimental setup: 1) Induction motor, 2) Inverter modules, 3) DSP and 4) Oscilloscope.

The gate driver signals are applied to the gates of the power switches. SKM100GB12T4 IGBT from SEMIKRON is used as the power switch. The experiment is performed on a 3-phase induction motor having rating: $5 \mathrm{~kW}, 200 \mathrm{~V}, 50 \mathrm{~Hz}, 4$ pole. The photograph of laboratory experimental prototype is shown in Fig. 4.

\section{B. Steady state results}

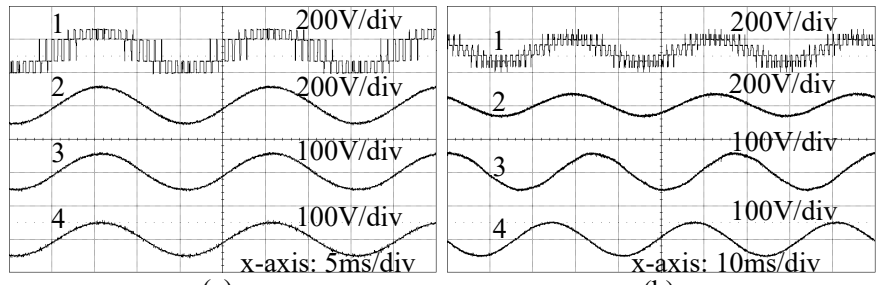

(a)

(b)

Fig. 5. Steady state operation on induction motor load: a) $50 \mathrm{~Hz}$ and b) $30 \mathrm{~Hz}$. Traces: 1) Phase voltage $\left(V_{A n}\right)$, 2) Fundamental component of phase voltage, 3) Fundamental component of first phase shifted notched waveform and 4) Fundamental component of second phase shifted notched waveform.

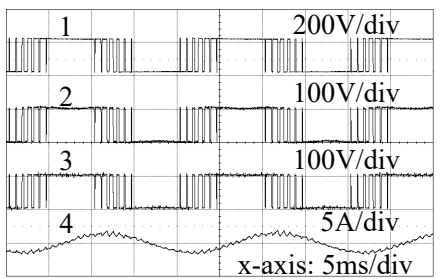

(a)

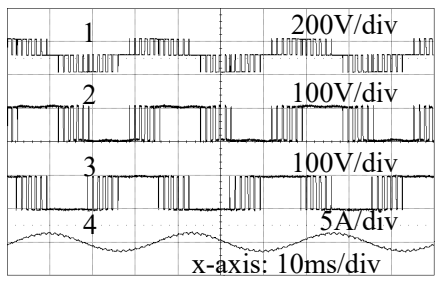

(b)
Fig. 6. Steady state operation on induction motor load: a) $50 \mathrm{~Hz}$ and b) $30 \mathrm{~Hz}$. Traces: 1) Pole voltage $\left.\left(V_{A O}\right), 2\right)$ First phase shifted notched waveform $\left(V_{A 1, \text { notch }}\right)$ 3) Second phase shifted notched waveform $\left(V_{A 2, \text { notch }}\right)$ and 4) Phase current $\left(I_{A}\right)$.

The steady state operation of the inverter with induction motor load at $50 \mathrm{~Hz}$ (base speed) and $30 \mathrm{~Hz}$ are shown in Fig. 5-7. Here, V/f ratio is maintained constant at both frequencies of $50 \mathrm{~Hz}$ and $30 \mathrm{~Hz}$. Since V/f ratio is maintained, the inverter delivers maximum fundamental voltage at $50 \mathrm{~Hz}$ (base speed). At this frequency of operation, to extract maximum fundamental component, there is no phase shift between the phase shifted waveforms (Fig. 5a Trace 3, 4 and Fig. 6a Trace 


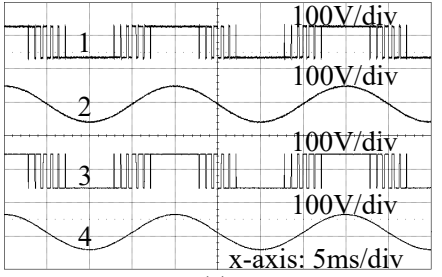

(a)

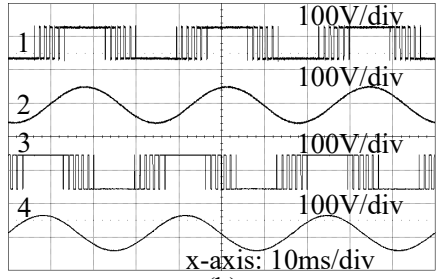

(b)
Fig. 7. Steady state operation on induction motor load: a) $50 \mathrm{~Hz}$ and b) $30 \mathrm{~Hz}$ Traces: 1) First phase shifted notched waveform $\left(V_{A 1, \text { notch }}\right)$, 2) Fundamental component of first phase shifted notched waveform, 3) Second phase shifted notched waveform $\left(V_{A 2, \text { notch }}\right)$ and 4) Fundamental component of second phase shifted notched waveform

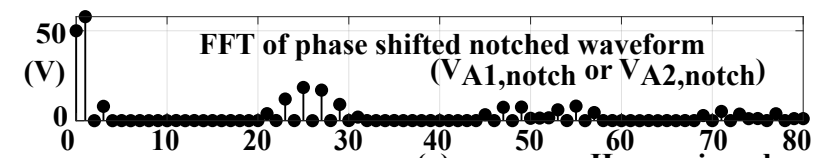

(a)

Harmonic order
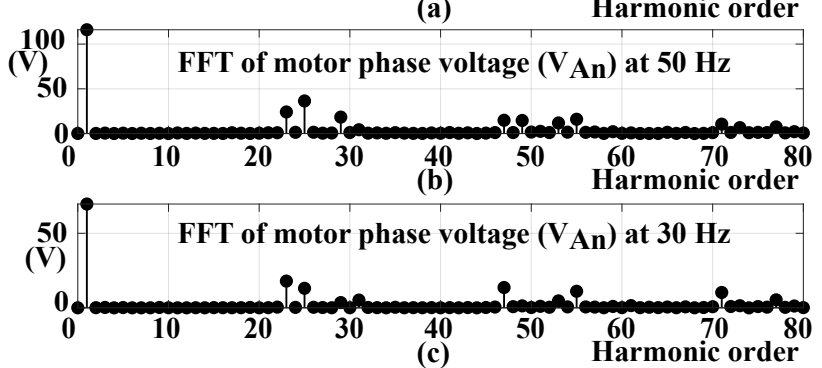

Fig. 8. Harmonic spectrum of: a) phase shifted notched waveform $\left(V_{A 2, \text { notch }}\right.$ or $\left.V_{A 2, \text { notch }}\right)$, b) Motor phase voltage at $50 \mathrm{~Hz}$ and c) Motor phase voltage at $30 \mathrm{~Hz}$ (For a DC-link voltage of 200V)

2, 3). So the pole voltage at $50 \mathrm{~Hz}$ (Fig. 6a Trace 1) which is the sum of two phase shifted notched waveforms $\left(V_{A O}=\right.$ $\left.V_{A 1, \text { notch }}+V_{A 2, \text { notch }}\right)$ has the same profile as that of the phase shifted waveforms (Fig. 6 Trace 3,5) but scaled by a factor of two. The fundamental and harmonic content in motor phase voltage $\left(V_{A n}\right)$ will be twice the corresponding fundamental and harmonic content of the phase shifted notched waveform $\left(V_{A 1, \text { notch }}\right.$ or $\left.V_{A 2, \text { notch }}\right)$, except for the triplen harmonics (which will be zero). This is also verified from the harmonic spectrum shown in Fig. 8a, b. This implies that the phase voltage at $50 \mathrm{~Hz}$ has a fundamental component of $116.4 \mathrm{~V}$ $\left(=0.582 V_{d c}\right)$, which is twice the fundamental component of notched waveform $\left(58.2 \mathrm{~V}=0.582 * V_{d c} / 2\right)$. It should be noted that this fundamental component $\left(0.582 V_{d c}\right)$ is higher than the fundamental component obtained in space vector PWM $\left(0.577 V_{d c}\right)$ in linear modulation. In addition, since the phase shifted notched waveform does not contain lower order harmonics till $19^{\text {th }}$ order, the resultant waveform is also devoid of these harmonics (see Fig. 8a, b).

At $30 \mathrm{~Hz}$ of operation, motor phase voltage has a fundamental component of $69.8 \mathrm{~V}\left(=(30 / 50) * 0.582 V_{d c}\right)$ to maintain the constant V/f ratio. Here the phase shifted waveforms (Fig. $5 \mathrm{~b}$ Trace 3, 4 and Fig. 7c) are shifted by $53.13^{\circ}\left(=\cos ^{-1}(30 / 50)\right)$ and $-53.13^{\circ}$ with respect to the fundamental component of the motor phase voltage (Fig. 5b Trace 2). It can also be noted that the profile and amplitude of the phase shifted notched waveforms $\left(V_{A 1, \text { notch }}\right.$ and $V_{A 2, \text { notch }}$ in Fig. 6 Trace 2,3) remains same irrespective of the frequency of operation and the required fundamental component in motor phase voltage is obtained by the phase shift between them. Fig. $8 \mathrm{c}$ shows the harmonic spectrum of motor phase voltage at $30 \mathrm{~Hz}$. Here also it can be seen that all the predefined lower order harmonics (till $19^{\text {th }}$ order) are eliminated from motor phase voltage. The phase current obtained during inverter operation at steady state of $50 \mathrm{~Hz}$ and $30 \mathrm{~Hz}$ is shown in Fig. 6. Since motor phase voltage does not contain lower order harmonics, these harmonics are also absent in the phase current resulting in mitigation of ill effects caused by lower order harmonic components in current.

\section{Transient state results}

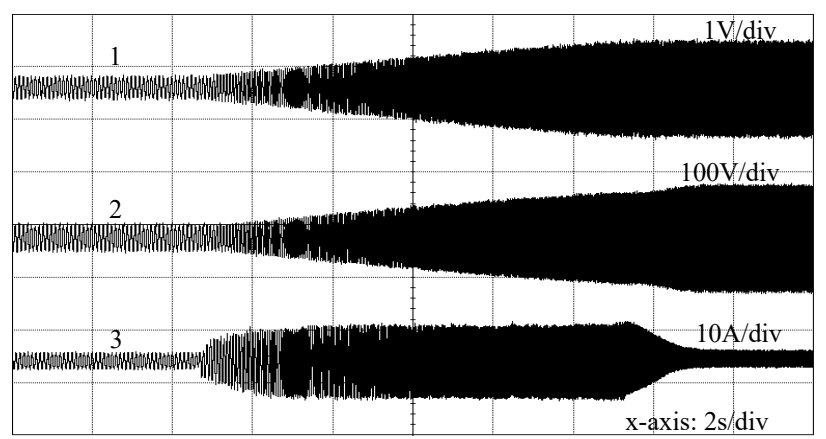

Fig. 9. Acceleration of the motor from $10 \mathrm{~Hz}$ to $50 \mathrm{~Hz}$ on induction motor load. Traces: 1) Modulating signal $\left.\left(V_{s}\right), 2\right)$ Phase voltage $\left(V_{A n}\right)$ and 3) Phase current $\left(I_{A}\right)$.

The results for the acceleration of the motor under constant V/f from $10 \mathrm{~Hz}$ to $50 \mathrm{~Hz}$ is shown in Fig. 9. A smooth transient is obtained during acceleration as any modulation index can be achieved by the scheme by proper adjustment of phase shift between the phase shifted notched waveforms.

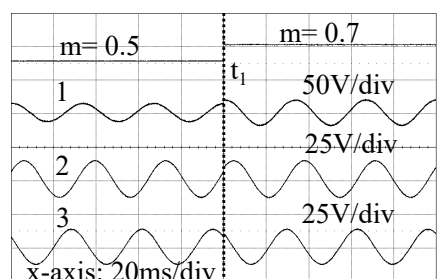

(a)

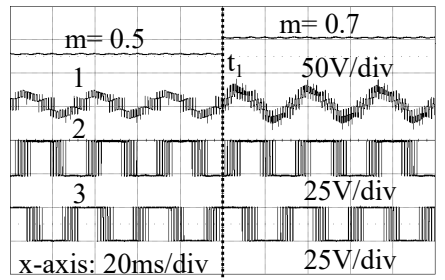

(c)

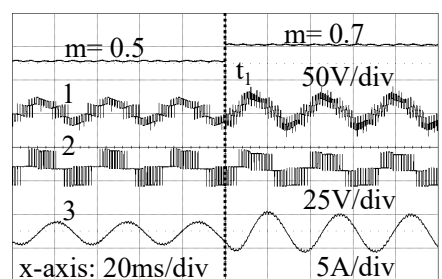

(b)

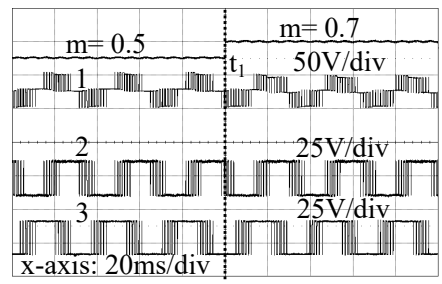

(d)
Fig. 10. Step change in modulation index (m) from 0.5 to 0.7 keeping frequency constant at $30 \mathrm{~Hz}$ on an RL load. a) Traces: 1) Fundamental component of phase voltage, 2) Fundamental component of first phase shifted notched waveform and 3) Fundamental component of second phase shifted notched waveform. b) Traces: 1) Phase voltage $\left.\left(V_{A n}\right), 2\right)$ Pole voltage $\left(V_{A O}\right)$ and 3) Phase current $\left(I_{A}\right)$. c) Traces: 1) Phase voltage $\left.\left(V_{A n}\right), 2\right)$ First phase shifted notched waveform $\left(V_{A 1, \text { notch }}\right)$ and 3$)$ Second phase shifted notched waveform $\left(V_{A 2, \text { notch }}\right)$. d) Traces: 1) Pole voltage $\left.\left(V_{A O}\right), 2\right)$ First phase shifted notched waveform $\left(V_{A 1, \text { notch }}\right)$ and 3) Second phase shifted notched waveform $\left(V_{A 2, \text { notch }}\right)$. 
To check the fast response of the scheme, a step change is given in modulation index and frequency of operation in Fig. 10 and Fig. 11 respectively. Since these fast response results cannot be taken on an induction motor (due to mechanical inertia), these results are taken on an RL load. In Fig. 10, a step change in modulation index $(\mathrm{m}$, where $\mathrm{m}=1$ for a fundamental component of $0.582 V_{d c}$ ) from 0.5 to 0.7 is introduced at $\mathrm{t}_{1}$ time instant at $30 \mathrm{~Hz}$ of operation with RL load. It can be seen that the scheme responds instantaneously (after a sampling duration of $20 \mu \mathrm{s}(=1 / 50 \mathrm{kHz}))$ to the reference command. The fundamental component of phase voltage (Fig. 10 Trace 1) changes to the new fundamental value at $t_{1}$ by adjusting the phase shift between the (Fig. 10a,c,d Trace 2,3) phase shifted notched waveforms. This experiment shows the quick responsiveness of the system for a step change in modulation index.

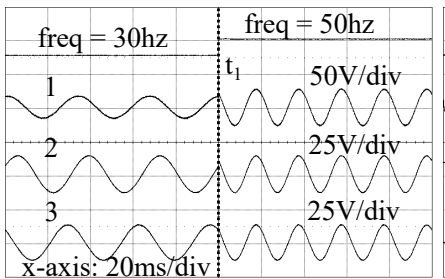

(a)

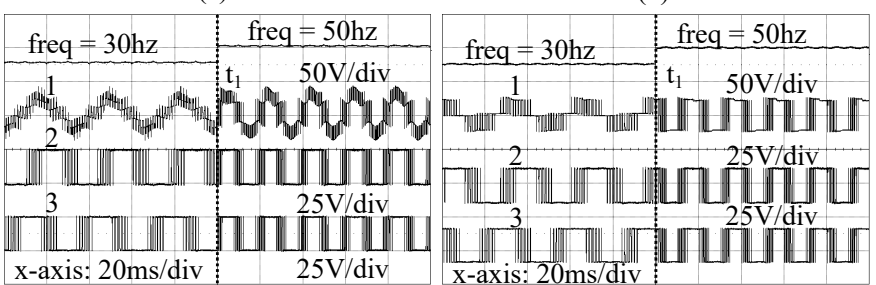

(c)

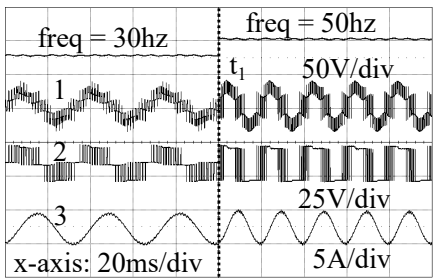

(b)

(d)
Fig. 11. Step change in operating frequency from $30 \mathrm{~Hz}$ to $50 \mathrm{~Hz}$ keeping V/f constant on an RL load. a) Traces: 1) Fundamental component of phase voltage, 2) Fundamental component of first phase shifted notched waveform and 3) Fundamental component of second phase shifted notched waveform. b) Traces: 1) Phase voltage $\left.\left(V_{A n}\right), 2\right)$ Pole voltage $\left(V_{A O}\right)$ and 3) Phase current $\left(I_{A}\right)$. c) Traces: 1) Phase voltage $\left(V_{A n}\right)$, 2) First phase shifted notched waveform $\left(V_{A 1, \text { notch }}\right)$ and 3$)$ Second phase shifted notched waveform $\left(V_{A 2, n o t c h}\right)$. d) Traces: 1) Pole voltage $\left(V_{A O}\right)$, 2) First phase shifted notched waveform $\left(V_{A 1, \text { notch }}\right)$ and 3) Second phase shifted notched waveform $\left(V_{A 2, \text { notch }}\right)$.

A step change in frequency of operation on an RL load while keeping the V/f ratio constant is investigated in Fig. 11. At time instant $t_{1}$, a step change from $30 \mathrm{~Hz}$ to $50 \mathrm{~Hz}$ is introduced. It can be seen from Fig. 11a Trace 1 that, as soon as the step command is given (after a sampling duration of $20 \mu \mathrm{s}$ ), the amplitude and frequency of the phase voltage changes. The change in amplitude and frequency of the phase voltage is achieved by variation of phase shift and frequency of the phase shifted notched waveforms $\left(V_{A 1, \text { notch }}\right.$ and $V_{A 2, \text { notch }}$ ) (see Fig. 11a,c,d Trace 2,3).

\section{CONCLUSION}

In this paper, a novel selective harmonic elimination method utilizing the same set of notch angles throughout the modulation range is proposed. At first, two identical notched square waveforms with predefined notches eliminating the predefined harmonics are generated. These waveforms are appropriately phase shifted and added to control the fundamental component to the desired value at the required frequency. Since predefined harmonics are eliminated in the notched waveforms, the resultant phase voltage are also devoid of these lower order harmonics irrespective of the frequency of operation. In addition, the linear modulation range is extended till 0.582 times the DC-link voltage in the proposed scheme in comparison with 0.577 times the DC-link voltage in the case of space vector PWM. The scheme requires minimum computation efforts as the phase shift is calculated in every sampling instant using simple algebra. In addition, the scheme doesn't require any initial guess, iterations or look up table to store the notch angle information for different modulation indices. Since the scheme calculates the phase shift at every sampling interval, the dynamics for step change in modulation index and frequency are almost instantaneous. The experimental results shows the efficacy of the scheme with the elimination of the predefined lower order harmonics.

\section{REFERENCES}

[1] D. Wang, Z. J. Shen, X. Yin, S. Tang, X. Liu, C. Zhang, J. Wang, J. Rodriguez, and M. Norambuena, "Model predictive control using artificial neural network for power converters," IEEE Transactions on Industrial Electronics, pp. 1-1, 2021.

[2] J. Guo, Y. Chen, S. Liao, W. Wu, X. Wang, and J. M. Guerrero, "Lowfrequency oscillation analysis of vsms-based vsc-hvdc systems based on the five-dimension impedance stability criterion," IEEE Transactions on Industrial Electronics, pp. 1-1, 2021.

[3] G. Gontijo, T. Kerekes, D. Sera, M. Ricco, L. Mathe, and R. Teodorescu, "Medium-voltage converter solution with modular multilevel structure and decentralized energy storage integration for high-power wind turbines," IEEE Transactions on Power Electronics, pp. 1-1, 2021.

[4] A. Tcai, Y. Kwon, S. Pugliese, and M. G. Liserre, "Reduction of the circulating current among parallel npc inverters," IEEE Transactions on Power Electronics, pp. 1-1, 2021.

[5] Y. Tang, Y. He, F. Wang, and R. Kennel, "Voltage-sourced converter fed high-speed srm drive system with energy feedback and near-unity power factor," IEEE Transactions on Industrial Electronics, pp. 1-1, 2021.

[6] S. I. Guggari, "Analysis of thermal performance metrics-application to cpu cooling in hpc servers," IEEE Transactions on Components, Packaging and Manufacturing Technology, vol. 11, no. 2, pp. 222-232, 2021.

[7] M. Wu, C. Xue, Y. R. Li, and K. Yang, "A generalized selective harmonic elimination pwm formulation with common-mode voltage reduction ability for multilevel converters," IEEE Transactions on Power Electronics, pp. 1-1, 2021.

[8] M. A. Memon, M. D. Siddique, M. Saad, and M. Mubin, "Asynchronous particle swarm optimization-genetic algorithm (apso-ga) based selective harmonic elimination in a cascaded h-bridge multilevel inverter," IEEE Transactions on Industrial Electronics, pp. 1-1, 2021.

[9] M. Wu, Y. W. Li, and G. Konstantinou, "A comprehensive review of capacitor voltage balancing strategies for multilevel converters under selective harmonic elimination pwm," IEEE Transactions on Power Electronics, vol. 36, no. 3, pp. 2748-2767, 2021.

[10] M. Wu, K. Wang, K. Yang, G. Konstantinou, Y. W. Li, and Y. Li, "Unified selective harmonic elimination control for four-level hybridclamped inverters," IEEE Transactions on Power Electronics, vol. 35, no. 11, pp. $11488-11501,2020$.

[11] A. Pérez-Basante, S. Ceballos, G. Konstantinou, J. Pou, I. Kortabarria, and I. M. d. Alegría, "A universal formulation for multilevel selectiveharmonic-eliminated pwm with half-wave symmetry," IEEE Transactions on Power Electronics, vol. 34, no. 1, pp. 943-957, 2019.

[12] J. R. Wells, X. Geng, P. L. Chapman, P. T. Krein, and B. M. Nee, "Modulation-based harmonic elimination," IEEE Transactions on Power Electronics, vol. 22, no. 1, pp. 336-340, 2007.

[13] C. Buccella, C. Cecati, M. G. Cimoroni, and K. Razi, "Analytical method for pattern generation in five-level cascaded h-bridge inverter using selective harmonic elimination," IEEE Transactions on Industrial Electronics, vol. 61, no. 11, pp. 5811-5819, 2014. 
[14] A. Janabi, B. Wang, and D. Czarkowski, "Generalized chudnovsky algorithm for real-time pwm selective harmonic elimination/modulation: Two-level vsi example," IEEE Transactions on Power Electronics, vol. 35, no. 5, pp. 5437-5446, 2020.

[15] K. Yang, Q. Zhang, R. Yuan, W. Yu, J. Yuan, and J. Wang, "Selective harmonic elimination with groebner bases and symmetric polynomials," IEEE Transactions on Power Electronics, vol. 31, no. 4, pp. 2742-2752, 2016.

[16] K. Yang, M. Feng, Y. Wang, X. Lan, J. Wang, D. Zhu, and W. Yu, "Realtime switching angle computation for selective harmonic control," IEEE Transactions on Power Electronics, vol. 34, no. 8, pp. 8201-8212, 2019.

[17] K. Yang, Q. Zhang, J. Zhang, R. Yuan, Q. Guan, W. Yu, and J. Wang, "Unified selective harmonic elimination for multilevel converters," IEEE Transactions on Power Electronics, vol. 32, no. 2, pp. 1579-1590, 2017.

[18] A. Guellal, C. Larbes, D. Bendib, L. Hassaine, and A. Malek, "Fpga based on-line artificial neural network selective harmonic elimination pwm technique," International Journal of Electrical Power and Energy Systems, vol. 68, pp. 33-43, 2015.

[19] P. Sanjeevikumar, C. Dhanamjayulu, and B. Khan, "Artificial neural network and newton raphson (ann-nr) algorithm based selective harmonic elimination in cascaded multilevel inverter for pv applications," IEEE Access, pp. 1-1, 2021.

[20] F. Filho, H. Z. Maia, T. H. A. Mateus, B. Ozpineci, L. M. Tolbert, and J. O. P. Pinto, "Adaptive selective harmonic minimization based on anns for cascade multilevel inverters with varying dc sources," IEEE Transactions on Industrial Electronics, vol. 60, no. 5, pp. 1955-1962, 2013.

[21] J. Wang and D. Ahmadi, "A precise and practical harmonic elimination method for multilevel inverters," IEEE Transactions on Industry Applications, vol. 46, no. 2, pp. 857-865, 2010.

[22] D. Ahmadi, K. Zou, C. Li, Y. Huang, and J. Wang, "A universal selective harmonic elimination method for high-power inverters," IEEE Transactions on Power Electronics, vol. 26, no. 10, pp. 2743-2752, 2011.

[23] M. Balasubramonian and V. Rajamani, "Design and real-time implementation of shepwm in single-phase inverter using generalized hopfield neural network," IEEE Transactions on Industrial Electronics, vol. 61, no. 11, pp. 6327-6336, 2014. 